\title{
NATURALISMO Y NORMATIVIDAD EN LA CRÍTICA PEIR- CEANA AL PSICOLOGISMO EN LÓGICA
}

\author{
Evelyn Vargas \\ Universidad Nacional de La Plata \\ Consejo Nacional de Investigaciones Científicas y Técnicas \\ (Argentina) \\ evargas@fahce.unlp.edu.ar \\ evelyn.vargas@gmail.com
}

\begin{abstract}
Resumen: El "pragmaticismo" de Peirce se propuso conciliar una concepción falibilista y naturalista de la racionalidad con su concepción inferencialista de la cognición. En este artículo, mi objetivo es analizar dicha perspectiva a la luz de sus críticas a las posiciones de los lógicos alemanes, quienes, de acuerdo con Peirce, han difundido una concepción falaz del razonamiento que tiene por consecuencia borrar la distinción entre razonamientos correctos e incorrectos. Si, como éstos sostendrían, la corrección lógica se limita al reconocimiento de la evidencia de la conclusión por parte de quien razona, el problema se reduce a una cuestión de la psicología. Peirce sostiene que el error de los lógicos alemanes se basa en que éstos "falsean los hechos del razonamiento (EP2: 243)," esto es, que los conciben erróneamente, y no en apelar a hechos para dar cuenta de la validez. Sin embargo, también subraya que la cuestión acerca del razonamiento correcto o incorrecto muestra el carácter normativo de la lógica; si se atiende correctamente a los fenómenos del razonamiento, éstos ponen de manifiesto que quien razona juzga que la inferencia está gobernada por una regla que conducirá a la verdad.
\end{abstract}

Palabras claves: Peirce, Pragmatismo, Naturalismo, Inferencia, Psicologismo, Regla

\begin{abstract}
Peirce's "pragmaticism" aims to conciliate a fallibilist and naturalist view on rationality with an inferentialist conception of cognition. In this paper my purpose is to analyze his approach in the light of his criticism to the German logicians who, according to him, have promoted a fallacious conception of reasoning that eliminates the distinction between good and bad reasoning. If logical validity consists in the recognition of the evidence of the conclusion by the reasoner, the problem would be tantamount to a problem of psychology. Their mistake, according to Peirce, results from falsifying the facts of reasoning (EP 2: 243), rather than from appealing to facts in order to account for logical correctness. However, he also emphasizes that the question concerning good or bad reasoning shows that logic is a normative science, that is, if the true phenomena of reasoning are taking into account, these show that the reasoner judges that her inference follows a rule that leads to the truth.
\end{abstract}

Key words: Peirce, Pragmatism, Naturalism, Inference, Psychologism, Rule 


\section{Introducción:}

Es bien conocido que el filósofo americano compartía con otros pragmatistas su adhesión al naturalismo. A grandes rasgos, las diferentes variantes naturalistas sostienen que las investigaciones empíricas que llevan a cabo las ciencias naturales son relevantes para las teorías filosóficas acerca del conocimiento, la racionalidad y otros problemas de la llamada epistemología tradicional. Ciertamente, Peirce creía que las investigaciones dentro de una disciplina pueden ser pertinentes para otras ciencias. Pero a diferencia de otros naturalistas, para quienes las cuestiones epistemológicas pueden responderse mediante los procedimientos descriptivos de las ciencias empíricas, él defendió con insistencia la dimensión normativa del conocimiento. Diferentes teorías de la racionalidad coinciden en conceder a la lógica un rol normativo respecto de nuestras creencias; un argumento válido, afirman, debe poder restringir las actitudes doxásticas de un agente racional. Por ejemplo, las normas de implicación o de consistencia regulan la formación o revisión de creencias, pues el agente no debe creer las premisas de un argumento válido sin creer al mismo tiempo en la conclusión, o sostener un conjunto de creencias inconsistentes. Pero el tipo de relación que caracteriza el vínculo entre la noción de validez y su rol en nuestras actitudes doxásticas es terreno de arduo debate. En un extremo encontramos partidarios del rol constitutivo de la lógica, mientras, en el otro extremo, algunos niegan cualquier relación entre las reglas lógicas y las normas de revisión de creencias, y finalmente algunos sostienen que este rol normativo puede esclarecer el concepto de validez.

Peirce y Dewey adhirieron al evolucionismo, y compartieron una concepción de la racionalidad humana "en un estado de insipiencia, de crecimiento (EP 2: 255). Sin embargo, el primero se mostró crítico de la posición del segundo tanto públicamente como en la correspondencia entre ambos. Un elemento común a dichas críticas es la insistencia de Peirce en que la concepción de Dewey de la lógica como una historia natural del pensamiento suprime el carácter normativo de la lógica. Así lo expresa en 1904 cuando publica una reseña de la obra de Dewey, "Studies in Logical Theory", en la que discute la concepción de éste. ${ }^{1}$ Es de notar que Peirce afirma allí que encuentra difícil distinguir la posición de Dewey de aquella sostenida por los lógicos alemanes de su

\footnotetext{
${ }^{1}$ Junto con la reseña para "The Nation," los "Collected Papers" incluyen fragmentos de la correspondencia con Dewey de 1904 y 1905 en los que Peirce intenta ampliar o incluso corregir lo escrito en la reseña.
} 
tiempo. ${ }^{2}$ Para Peirce los lógicos alemanes son culpables de haber desatado una "enfermedad" que pone en riesgo la vida misma de la ciencia. Su culpa reside en haber difundido una concepción falaz del razonamiento que tiene por consecuencia borrar la distinción entre razonamientos correctos e incorrectos. Si, como éstos sostendrían, la corrección lógica se limita al reconocimiento de la evidencia de la conclusión por parte de quien razona, el problema se reduce a una cuestión de la psicología. Esta crítica sugiere que Peirce entiende la normatividad de la lógica en términos de la universalidad y necesidad de las reglas lógicas. Pero no establece de suyo un vínculo entre la noción de validez y el rol de la lógica en nuestros sistemas de creencias, y más específicamente, la relevancia de esto último para la noción de validez.

En lo que sigue sostendré que en su crítica a la forma de psicologismo en lógica representado por los lógicos alemanes es posible hallar la convergencia de aquellas dos tendencias de su pensamiento que señalé al comienzo. Como veremos a continuación, Peirce sostiene que el error de los lógicos alemanes se basa en que éstos "falsean los hechos del razonamiento (EP2: 243)," esto es, que los conciben erróneamente, y no en apelar a hechos para dar cuenta de la validez. Sin embargo, también subraya que la cuestión acerca del razonamiento correcto o incorrecto muestra el carácter normativo de la lógica. Más aun, si se atiende correctamente a los fenómenos del razonamiento, éstos ponen de manifiesto que quien razona juzga que la inferencia está gobernada por una regla que conducirá a la verdad. Es precisamente este juicio que acompaña al proceso de inferencia el que distingue la inferencia lógica de una mera asociación de ideas.

\section{La falacia:}

\subsection{La inferencia como modo de adquisición de creencias:}

Es importante señalar desde un comienzo que la objeción de Peirce no se limita a cuestiones técnicas relativas a la lógica formal tal como hoy se la entiende; podemos decir que Peirce es un inferencialista en el sentido de que entiende que toda cognición es un proceso inferencial, ${ }^{3}$ Mediante nuestros procesos inferenciales formamos nuevas creencias a partir de nuestras creencias previas; cuando la inferencia es válida, podemos formar creencias verdaderas a partir de nuestras creencias conocidas previas. Más precisamente,

\footnotetext{
${ }^{2}$ Véase por ejmplo, CP 8.190

${ }^{3}$ Es conocida la explicacación que ofrece tempranamente en los artículos de la llamada "Cognition Series."
} 
su caracterización de la inferencia apela a este rol en nuestras creencias, y así lo señala en numerosas ocasiones. Escribe Peirce en diferentes momentos:

But it is a matter of constant experience, that if a man is made to believe in the premises, in the sense that he will act from them and will say that they are true, under favorable conditions he will also be ready to act from the conclusion and to say that that is true. W 2: 214

Reasoning is accepting a proposition as true, while recognizing some other proposition as the reason for it. By recognizing a proposition as the reason for another, is meant recognizing that the belief in the former causes the belief in the latter in a way in which true propositions will not, (at least usually,) produce belief in such as are false. W 4: 245

Reasoning is the process by which we attain a belief which we regard as the result of previous knowledge. EP 2: 11

Puesto que la caracterización del razonamiento en términos de formación de creencias a partir de otras no se pronuncia acerca del alcance de la creencia concluida, este modo de entender el razonamiento puede extenderse a las tres formas de inferencia que Peirce admite, esto es, la deducción, la inducción y la abducción. ${ }^{4}$ No obstante, en lo que sigue me limitaré al caso de la deducción, esto es, al razonamiento necesario. ${ }^{5}$

En el último pasaje citado Peirce enfatiza que el punto de partida de una inferencia son otras creencias conocidas. Ahora bien, si no es posible dar cuenta de la validez de los procesos inferenciales, se pone en cuestión toda forma de adquisición y justificación de conocimientos; de allí la importancia que Peirce adjudica a la falacia de los lógicos alemanes. Por otro lado, el reconocimiento de este rol normativo de las inferencias válidas respecto de nuestras creencias no dice nada acerca de la importancia que pudiera tener este rol para esclarecer la noción de validez, si lo tuviera. Sin embargo, al aceptar que un argumento es válido, nos sometemos a una norma por la cual no debemos creer las premisas sin creer al mismo tiempo la conclusión, de modo que este rol restrictivo respecto de nuestras creencias puede servir para esclarecer la noción de validez sin limitarnos a un sistema lógico en particular. ${ }^{6}$ Tal vez podríamos decir que el hecho de que cumple esta

\footnotetext{
${ }^{4}$ véase, por ejemplo: If we are to give the names of Deduction, Induction, and Abduction to the three grandnclasses of inference, then Deduction must include every attempt at mathematical demonstration, whether it relate to single occurrences or to "probabilities," that is, to statistical ratios; Induction must mean the operation that induces an assent, with or without quantitative modification, to a proposition already put forward, this assent or modified assent being regarded as the provisional result of a method that must ultimately bring the truth to light; while Abduction must cover all the operations by which theories and conceptions are engendered. CP 5.590

${ }^{5}$ Como explica Peirce: If we can state wherein the validity of deductive resoning lies, we shall have defined the foundation of logical goodness of whatever kind. (EP 2: 206)

${ }^{6}$ En la discusión contemporánea Hartry Field ha defendido una posición similar.
} 
función respecto de nuestras creencias permite hacer inteligible la relación entre la lógica (entendida en sentido restringido) y nuestras actitudes doxásticas, de modo que permita resolver conflictos normativos sin apelar simplemente a la verdad de los principios lógi$\cos$.

\subsection{La inferencia según los lógicos alemanes:}

Como dijimos, Peirce identifica el argumento a favor del psicologismo que defenderían los lógicos alemanes con una concepción errónea del razonamiento; más precisamente, estos lógicos conciben erróneamente "los hechos del razonamiento (EP2: 243)," y por consiguiente, destruyen la distinción entre razonamiento correcto e incorrecto. ${ }^{7} \mathrm{El}$ texto en el que Peirce desarrolla con más detalle esta crítica corresponde a la primera de ocho conferencias que pronunció en el Instituto Lowell de Boston el 23 de noviembre de $1903,{ }^{8}$ el mismo año de sus célebres conferencias de Harvard sobre pragmatismo. ${ }^{9}$ La estrategia de Peirce consiste en formular el argumento que comparten aquellos que falsean los hechos del razonamiento, y luego refutarlo, de allí que lo denomine como la falacia del acusado, ${ }^{10}$ pues Peirce asume la carga de la prueba, como lo haría un fiscal en un proceso judicial. Establecer de qué modo los "acusados" falsean los hechos no requerirá una refutación formal exhaustiva sino confrontarlos con la descripción de los fenómenos que efectivamente se presentan cuando razonamos. En tanto dicha descripción es un asunto de la fenomenología, ${ }^{11}$ Peirce podría sostener que evita la circularidad tanto como la regresión en la que caen los intentos tradicionales de justificación de las reglas de inferencia mediante principios. ${ }^{12}$

También el argumento rechazado por Peirce buscaba evitar caer en alguna de estas objeciones escépticas clásicas al intentar hallar un término al proceso de justificación al apelar al "sentimiento lógico" (logisches Gefühl) que experimentamos al razonar. En

\footnotetext{
${ }^{7}$ Peirce se refiere a la cuestión en términos de razonamientos buenos malos, marcando la semejanza entre el razonamiento y la conducta ética, en tanto en ambos casos se trata de una conducta auto-controlada y deliberada, siguiendo una norma.

8 "Some Topics of Logic bearing on Questions now Vexed," en EP 2: 242-257.

9 "Harvard Lectures on Pragmatism", en EP 2: 133-241.

${ }^{10}$ en inglés, "defendant," que en el sistema jurídico desde el derecho romano se presume inocente hasta probarse su culpabilidad. También se emplea para refereirse al demandado en una causa civil.

11 O también "faneroscopía," la ciencia que caracteriza de este modo: a science that does not draw any distinction of good and bad in any sense whatsoever, but just contemplates phenomena as they are, simply opens it eyes and describes what it sees. (...) I will not restrict it to the observation and analysis of experience but extend it to describing all the features that are common to whatever is experienced or might conceivably be experienced or become an object of study in any way direct or indirect. EP 2: 143

${ }^{12}$ Este es el conocido mecanismo de los tropos de Agripa, que Lewis Carroll (y en nuestra lengua, Jorge Luis Borges) han descripto con gran dramatismo.
} 
otras palabras, la relación de consecuencia lógica se percibe como evidente o correcta por parte de quien razona. La falacia del "acusado" podría resumirse de la siguiente manera:

1. Todo razonamiento tiene lugar en una mente.

2. Si el razonamiento no satisface el "sentimiento lógico" de esa mente, no sería su razonamiento.

3. Si el razonamiento lo satisface, no sería criticable.

4. Ahora bien, solo podemos saber que el razonamiento es correcto por el sentimiento lógico: la crítica de un razonamiento se lleva a cabo mediante otro razonamiento, pero ese otro razonamiento debe aceptarse o rechazarse por el sentimiento lógico o por otro razonamiento. Pero no es posible llevar a cabo una serie sin fin de razonamientos.

5. Por tanto, el "acusado" debe aceptar las siguientes consecuencias:

6. Un razonamiento final debe adoptarse bajo el supuesto según el cual satisfacer el sentimiento es lo que hace a un razonamiento correcto. Pero todo razonamiento satisface el sentimiento lógico del que razona.

7. De allí se sigue la perniciosa consecuencia denunciada por Peirce:

8. No hay distinción entre razonamiento bueno o malo. ${ }^{13}$

Peirce no pone en cuestión la pertinencia de las primeras dos premisas; después de todo él también habría de apelar a lo que sucede cuando razonamos, ${ }^{14}$ pero encuentra objetable que la corrección de un razonamiento dependa de la convicción que sentimos cuando concluimos, por ejemplo, que Sócrates es mortal a partir de las consabidas premisas. La cuarta premisa muestra cómo el lógico falaz intenta escapar a la regresión apelando a algo extra-lógico, esto es, un sentimiento, que, por su misma naturaleza, no requiere justificarse mediante un razonamiento. La conclusión de Peirce se sigue justamente, del hecho de que tal sentimiento no puede someterse a crítica, y consecuentemente, no podría distinguirse entre un razonamiento correcto y otro que no lo es. El poder de persuasión de falacias como la de la afirmación del consecuente dan cuenta de este problema.

En la conferencia Peirce añade que la falacia de los lógicos alemanes depende de dos premisas que son falsas:

\footnotetext{
${ }^{13}$ Sigo la presentación en EP 2: 244

${ }^{14}$ Haack (1978) califica la posición de Peirce como una forma de psicologismo (238).
} 
a. Es imposible que una conclusión se extraiga a menos que esté acompañada de un sentimiento lógico.

b. Si todo razonamiento está determinado por nuestro sentimiento lógico, no puede haber distinción entre razonamientos buenos y malos.

Contra la segunda premisa, Peirce argumenta que, aunque se admita que un sentimiento lógico determina nuestros razonamientos, la distinción entre buenos y malos razonamientos depende de la comparación con normas. Juzgar que un razonamiento satisface tales normas no es equivalente a la sensación de satisfacción que acompaña dicho juicio. Pero por ello es la primera premisa a la que califica de monstruosa. Basar la corrección del razonamiento en lo que se nos presenta como un buen razonamiento es fundar la lógica en el supuesto de que un razonamiento que de hecho nos parece correcto, lo es. Y añade, sin más, que ... "el único hecho del que realmente depende la solidez de todo razonamiento y la verdad de todo pensamiento humano es que las conjeturas de un hombre son de algún modo mejores que proposiciones meramente al azar." (EP 2: 252, la traducción es propia). Aquí entendemos que una hipótesis es superior a otra en tanto es más adecuada para nuestros fines, que en el caso de la lógica es conducirnos más rápidamente a la verdad. ${ }^{15}$ Esta relación de la conducta llevada a cabo, esto es, efectuar un razonamiento, con alcanzar un fin permite establecer su semejanza con la conducta moral, en la que también interviene una norma, y la comparación de la conducta particular con dicha norma.

\section{La corrección lógica como cuestión de hecho:}

Desde un punto de vista histórico la crítica de Peirce puede situarse en el contexto de la disputa del psicologismo, según el término acuñado por Erdman en 1866, y que se desarrolló sobre todo en el mundo de habla alemana, entre 1890 y la primera guerra mundial. ${ }^{16}$ Sus detractores se caracterizaron por una actitud hostil hacia la capacidad de las ciencias naturales o sociales para dar respuesta a las cuestiones centrales de la lógica; las figuras más conocidas de la controversia fueron Frege y Husserl, pero J. S. Mill también

\footnotetext{
${ }^{15}$ Dice Peirce: a question of what is good reasoning and what bad is not a question of whether the mind approves it or not, but is a question of fact. A method that tends to carry us toward the truth more speedily than we could otherwise progress is good, ... EP 2: 252

${ }^{16}$ Para un interesante análisis contemporáneo de este debate véase Kusch (1995)
} 
rechazó el psicologismo. Se trata de una disputa acerca de la relación entre lógica y psicología, y distintas variaciones pueden caracterizarse como psicologistas. De entre éstas, la posición criticada por Peirce se centra en la supuesta auto evidencia de la que gozarían los razonamientos; este sentimiento de evidencia, en tanto es una forma de la experiencia, haría de la lógica una rama de la psicología. Pero, sostiene Peirce, el conocimiento de los procesos de pensamiento es irrelevante para establecer la naturaleza del razonamiento. ${ }^{17}$

Al mismo tiempo, como señalamos en la sección precedente, Peirce apela a los fenómenos que tienen lugar cuando razonamos para refutar la falacia. Según Peirce la inferencia tiene tres pasos esenciales, que denomina coligación, observación, y juicio, respectivamente (EP 2: 24; 213). En el primero se reúnen las proposiciones que forman las premisas, su representación conforma un ícono complejo cuya observación sugiere otro, la conclusión.

El primer paso de un razonamiento necesario consiste en considerar juntas las premisas, es decir, las proposiciones tenidas por verdaderas por parte de quien infiere. Pero sea que aquel que razona considere una única premisa (razonamiento inconsciente) o una conjunción de ellas, se forma un ícono complejo, y el paso siguiente consiste en observar dicho ícono (EP 2: 22). La acción de contemplar este diagrama es un acto de observación pues, al igual que en la percepción sensible, lo percibido limita el contenido representado, (por ejemplo, en condiciones normales, no me es posible alterar voluntariamente la imagen sensible, y ver como rojo un árbol verde sin que deje de ser una percepción). De la misma manera, quien razona se encuentra restringido por lo que percibe en el ícono o diagrama. Se trata más propiamente de una observación, pues en la percepción que llamamos observación se añade el reconocimiento deliberado de esta restricción $\left(\right.$ CP 5. 582). ${ }^{18}$

Los diagramas son íconos de las formas de las relaciones que constituyen el objeto, y por esta razón, piensa Peirce, pueden mostrar un “deber-ser.” El ejemplo elegido por Peirce para ilustrar esto último proviene de las matemáticas; se trata de la prueba según la cual las relaciones entre los miembros de una colección son necesariamente más

\footnotetext{
${ }^{17}$ At any rate, a knowledge of the processes of thinking, even if it were at hand, would be entirely irrelevant to that sort of knowledge of the nature of our reasonings which it is incumbent upon us to have in order that we may give them our deliberate approval. CP 2.185

${ }^{18}$ Dice peirce: For what is observation? What is experience? It is the enforced element in the history of our lives. It is that which we are constrained to be conscious of by an occult force residing in an object which we contemplate. The act of observation is the deliberate yielding of ourselves to that force majeure -- an early surrender at discretion, due to our foreseeing that we must, whatever we do, be borne down by that power, at last. CP 5.581
} 
numerosos que el número de sus miembros. La prueba de Peirce se basa en un diagrama que representa una colección dada, en él quien razona ve “... que no hay relación posible en la cual cada colección involucrada (considerada como un objeto singular), se halle con respecto a un miembro de una colección dada, sin que otra de las colecciones involucradas se halle en la misma relación con respecto al mismo miembro de una colección dada."19 Otros ejemplos más familiares lo constituyen los diagramas de Venn para los razonamientos silogísticos, o las construcciones en geometría. ${ }^{20}$

La corrección lógica hace referencia a la propiedad de preservar la verdad que distingue el razonar de otras formas de combinación de creencias, y es esta relación entre las premisas y la conclusión la que ha de poderse observar en el ícono que la representa. De este modo, la observación que tiene lugar al razonar no es simplemente un dispositivo conveniente para facilitar la inferencia, sino una parte constitutiva de ella:

... reasoning consists in the observation that where certain relations subsist certain others are found, and it accordingly requires the exhibition of the relations reasoned within an icon. CP 3.363

Peirce describe la base del razonar como una "analogía completa" entre el tema del razonamiento y el ícono, esto es, las relaciones entre las partes del objeto se hallan representadas en el ícono o diagrama:

The truth, however, appears to be that all deductive reasoning, even simple syllogism, involves an element of observation; namely, deduction consists in constructing an icon or diagram the relations of whose parts shall present a complete analogy with those of the parts of the object of reasoning, of experimenting upon this image in the imagination, and of observing the result so as to discover unnoticed and hidden relations among the parts.

For instance, take the syllogistic formula,

All $\mathrm{M}$ is $\mathrm{P}$

$\mathrm{S}$ is $\mathrm{M}$

$\therefore \mathrm{S}$ is $\mathrm{P}$.

\footnotetext{
19 “... that there is no possible relation in which each involved collection (considered as a single object), stands to a member of a given collection, without any other of the involved collections standing in the same relation to that same member of the given collection (CP 4.532)."

${ }^{20}$ In mathematical reasoning there is a sort of observation. For a geometrical diagram or array of algebraical symbols is constructed according to an abstractly stated precept, and between the parts of such diagram or array certain relations are observed to obtain, other than those which were expressed in the precept. These being abstractly stated, and being generalized, so as to apply to every diagram constructed according to the same precept, give the conclusion. CP 2.216
} 
This is really a diagram of the relations of $\mathrm{S}, \mathrm{M}$, and $\mathrm{P}$. The fact that the middle term occurs in the two premisses is actually exhibited, and this must be done or the notation will be of no value. Ibid. (el énfasis es mío)

Tal como ocurre en la representación diagramática de un silogismo, en la que la conclusión se muestra, o en la construcción de una figura ciertos ángulos y líneas quedan representados en el análisis geométrico, en general cuando elegimos un diagrama que representa ciertas relaciones, otras relaciones también resultan determinadas, pero entendemos que estas otras relaciones se hallarán también en el objeto. Por esto Peirce dice que la analogía es completa. ${ }^{21}$ Ahora bien, las relaciones entre las partes de un razonamiento que el diagrama representa constituyen una norma o patrón inferencial. Mediante la representación icónica de las premisas se retrata la relación entre ellas y así queda trazada la conclusión. Es de notar que al igual que en el análisis geométrico su alcance excede al de la figura particular construida, de la misma manera, la dependencia observada en el diagrama es de naturaleza general puesto que se aplica a todos los casos similares:

Hence the mind is not only led from believing the premises to judge the conclusion true, but it further attaches to this judgment another that every proposition like the premise, that is having an icon like it, would involve, and compel acceptance, of a proposition related to it as the conclusion then drawn is related to that premise. EP 2: 24

Pero si bien la visualización por sí misma es una cuestión de creciente interés en la actualidad, nos interesada destacar con relación a nuestros propósitos presentes que, como señalamos al comienzo de esta sección, y se explicita en la cita precedente, Peirce añade un tercer paso junto a la coligación y la observación del diagrama, que debe poder acompañar el extraer la conclusión. En una inferencia en sentido estricto, una creencia se acepta de manera reflexiva y controlada; cuando formulamos una conclusión, se involucra el pensamiento según el cual toda inferencia que tenga la misma relación entre las premisas, será válida, esto es, preservará la verdad de la conclusión. Si faltara este juicio por parte de quien razona, la conclusión no sería tal sino una mera idea que se me presenta a la mente. ${ }^{22}$

\footnotetext{
${ }^{21}$ Nótese que la expresión 'analogía completa' es empleada por Kant en KU, y en una nota al parágrafo 84 de la denominada Lógica Jäsche, que Peirce conocía.

22 Par una comparación de la inferencia con la asociación de ideas, véase Vargas (2017).
} 
Dicho de otro modo, cuando juzgo que la inferencia que realicé es correcta, reconozco al mismo tiempo que la vinculación observada puede extenderse a nuevos casos aun no considerados. Para Peirce, entonces, la semejanza que entra en juego en el diagrama que representa la inferencia no es una mera asociación arbitraria, puesto que el ícono, el elemento de observación, representa las relaciones que pueden hallarse de modo que la conclusión no es una representación arbitraria.

En efecto, al juicio según el cual la conclusión es verdadera, se añade otro, que afirma que toda proposición que tiene un icono como las premisas no solo involucraría la conclusión sino que demandaría aceptar una proposición relacionada con ella tal como la conclusión se relaciona con ella. En ocasiones, Peirce señala que dicho juicio también es resultado de una inferencia. ${ }^{23} \mathrm{El}$ reconocimiento de este juicio pone en evidencia, según Peirce, que al pensar la inferencia, en tanto se considera como tal, se la reconoce en términos de una clase de inferencias. ${ }^{24}$ Ahora bien, esta regla general que se observa en el diagrama se representa con algún grado de vaguedad:

Thus we see, what is most important, that every inference is thought of, at the time of drawing it, as one of a possible class of inferences. In the case of a rational inference, we see, in an icon which represents the dependence of the icon of the conclusion upon the icon of the premise, about what that class of inference is, although as the outlines of icons are always more or less vague, thee is always more or less of vagueness in our conception of that class of inferences. Ibid.

Para que tenga lugar una inferencia, hemos de observar en el ícono que muestra la relación de la conclusión con las premisas, la regla que guía esa clase de inferencia. En la medida que toda representación icónica tiene algún grado de vaguedad respecto de lo

\footnotetext{
${ }^{23}$ Escribe Peirce: When the inference is first drawn, the leading principle is not present to the mind, but the habit it formulates is active in such a way that, upon contemplating the believed premiss, by a sort of perception the conclusion is judged to be true. Afterwards, when the inference is subjected to logical criticism, we make a new inference, of which one premiss is that leading principle of the former inference, according to which propositions related to one another in a certain way are fit to be premiss and conclusion of a valid inference, while another premiss is a fact of observation, namely, that the given relation does subsist between the premiss and conclusion of the inference under criticism; whence it is concluded that the inference was valid. CP 3.164

${ }^{24}$ Escribe Peirce: one finds that the diagram itself in its individuaity is not what the reasoning is concerned with (EP 2: 206) Y un poco más adelante en la misma conferencia: But in any case, either in the new diagram or else, in passing from one diagram to the other, the interpreter of the argumentation will be supposed to see something which will present this little difficulty for the theory of vision, that it is of a general nature. (...) all reasoning, so far as it depends on necessary reasoning, that is to say, mathematical reasoning, turns upon the perception of generality and continuity at every step (EP 2: 207).
} 
representado, habrá también vaguedad en la representación de la clase de inferencia. La representación icónica no muestra todos los casos posibles. ${ }^{25}$ Peirce entiende la generalidad en términos de potencialidad, tal como lo indica el uso del modo condicional en inglés, esto es, que toda proposición como las premisas involucraría (would involve) otra proposición como la conclusión. Pero, justamente por esto, la cuestión de si una inferencia es lógica o no, no debe confundirse con la cuestión de cómo nos sentimos cuando razonamos (EP 2: 166); más aun, no es posible sentir lo que sucedería sino solo lo que ha sucedido (EP 2: 168). Una norma, por el contrario, involucra los infinitos casos posibles que rige. Puede entenderse entonces la crítica de Peirce a los lógicos alemanes de su tiempo (su ejemplo habitual es Sigwart). La interpretación de la validez lógica que ellos proponen no puede dar cuenta de la bondad lógica de una inferencia pues ignora el hecho de que quien razona formula también un juicio acerca de su validez en el sentido de que establece su conformidad con un patrón inferencial, y éste consiste en una potencialidad que es representada, aunque vagamente, en el diagrama.

\section{Conclusiones}

El naturalismo al que ascribe Peirce no descarta la relevancia de las descripciones de los fenómenos que tienen lugar cuando razonamos; por el contrario, éstos nos permiten entender la corrección lógica en tanto cumple un rol prescriptivo respecto de nuestras actitudes doxásticas. Si aquella se entiende adecuadamente, involucra, no solo el seguimiento de una regla sino su reconocimiento, esto es, que quien razona juzga también que ésta se conforma a una relación entre las premisas que hacen verdadera otra proposición. Así la lógica cumple un rol normativo respecto de nuestras creencias, en tanto debemos creer en la conclusión si aceptamos las premisas, pero la noción de corrección lógica es naturalmente una noción normativa puesto que involucra una generalidad, la de los casos potencialmente incluidos en la regla que guía la inferencia, y que solo podemos conocer vagamente. Al vincular estas dos perspectivas sobre el rol normativo del razonamiento, Peirce pone en evidencia el carácter inacabado de la razón humana.

\section{Referencias}

\footnotetext{
${ }^{25}$ La noción de vaguedad tiene una extrema importancia para Peirce. Aquí nos limitamos a señalar que un signo vago es aquel que puede ser determinado mediante otros signos ( v. por ejemplo, EP 2, 350-53)
} 
Field, H. (2009). "What is the Normative Role of Logic?”, Proceedings of the Aristotelian Society, 83(1), 251-268.

Haack, S (1978). Philosophy of Logics, Cambridge, UK: Cambridge University Press.

Kant, I. Logik - Ein Handbuch zu Vorlesungen (im Auftrag Kants hrsg. von Gottlob Benjamin Jäsche) (trad. española: Lógica. Un manual de lecciones, Vázquez Lobeiras, M. (ed.), Madrid: Akal, 2000)

Kants gesammelte Schriften, herausgegeben von der Deutschen (formerly Königlichen Preussischen) Akademie der Wissenschaften, 29 vols., Berlin: Walter de Gruyter, 1902. $[=\mathrm{Ak}]$

Kusch, M. Psyhologism. A Case Study in the Sociology of Philosophical Knowledge, London - New York: Routledge.

Peirce, C. (1931-5; 1958). Collected Papers. Hartshorne, C. and Weiss, P. (eds. Vols.16); Burks, A. (eds. Vol. 8), Cambridge: Harvard University Press,. [= CP]

— (1998). The Essential Peirce: Selected Philosophical Writings. Houser, N. et alia (eds.) Bloomington: Indiana University Press, [=EP ]

— (1982- ...) Writings of Charles S. Peirce: A Chronological Edition, Fisch, M. Et alia (eds.) Volumes 1- 6; 8, Bloomington: Indiana University Press.

Vargas, E. (2017) “Perception as Inference," en Hull, K. \& Atkin, R. Peirce on Perceiving and Reasoning, London-New York: Routledge, , pp.14-24. 